\title{
Simultaneous EEG-fMRI at ultra-high field: Artifact prevention and safety assessment
}

\author{
João Jorge ${ }^{\mathrm{a}, \mathrm{b}, *}$, Frédéric Grouiller ${ }^{\mathrm{c}}$, Özlem Ipek ${ }^{\mathrm{d}}$, Robert Stoermer $^{\mathrm{e}}$, Christoph M. Michel ${ }^{\mathrm{f}}$, Patrícia Figueiredo ${ }^{\mathrm{a}}$, \\ Wietske van der Zwaag ${ }^{\mathrm{d}}$, Rolf Gruetter ${ }^{\mathrm{b}, \mathrm{g}, \mathrm{h}}$
}

a Institute for Systems and Robotics and Department of Bioengineering, Instituto Superior Técnico, University of Lisbon, Lisbon, Portugal

b Laboratory for Functional and Metabolic Imaging, École Polytechnique Fédérale de Lausanne, Lausanne, Switzerland

c Biomedical Imaging Research Center, Geneva University Hospital, University of Geneva, Geneva, Switzerland

d Biomedical Imaging Research Center-Animal Imaging and Technology, École Polytechnique Fédérale de Lausanne, Lausanne, Switzerland

e Brain Products, GmbH, Gilching, Germany

${ }^{\mathrm{f}}$ Department of Fundamental Neurosciences, University of Geneva, Geneva, Switzerland

${ }^{g}$ Department of Radiology, University of Lausanne, Lausanne, Switzerland

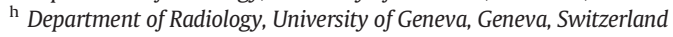

\section{A R T I C L E I N F O}

\section{Article history:}

Accepted 24 October 2014

Available online 29 October 2014

\section{Keywords:}

Simultaneous EEG-fMRI

Ultra-high field

Electromagnetic simulation

Visual evoked potential

Alpha wave

\begin{abstract}
A B S T R A C T
The simultaneous recording of scalp electroencephalography (EEG) and functional magnetic resonance imaging (fMRI) can provide unique insights into the dynamics of human brain function, and the increased functional sensitivity offered by ultra-high field fMRI opens exciting perspectives for the future of this multimodal approach. However, simultaneous recordings are susceptible to various types of artifacts, many of which scale with magnetic field strength and can seriously compromise both EEG and fMRI data quality in recordings above $3 \mathrm{~T}$. The aim of the present study was to implement and characterize an optimized setup for simultaneous EEG-fMRI in humans at $7 \mathrm{~T}$. The effects of EEG cable length and geometry for signal transmission between the cap and amplifiers were assessed in a phantom model, with specific attention to noise contributions from the MR scanner coldheads. Cable shortening (down to $12 \mathrm{~cm}$ from cap to amplifiers) and bundling effectively reduced environment noise by up to $84 \%$ in average power and $91 \%$ in inter-channel power variability. Subject safety was assessed and confirmed via numerical simulations of RF power distribution and temperature measurements on a phantom model, building on the limited existing literature at ultra-high field. MRI data degradation effects due to the EEG system were characterized via $\mathrm{B}_{0}$ and $\mathrm{B}_{1}^{+}$field mapping on a human volunteer, demonstrating important, although not prohibitive, $B_{1}$ disruption effects. With the optimized setup, simultaneous EEG-fMRI acquisitions were performed on 5 healthy volunteers undergoing two visual paradigms: an eyes-open/eyes-closed task, and a visual evoked potential (VEP) paradigm using reversing-checkerboard stimulation. EEG data exhibited clear occipital alpha modulation and average VEPs, respectively, with concomitant BOLD signal changes. On a single-trial level, alpha power variations could be observed with relative confidence on all trials; VEP detection was more limited, although statistically significant responses could be detected in more than $50 \%$ of trials for every subject. Overall, we conclude that the proposed setup is well suited for simultaneous EEG-fMRI at $7 \mathrm{~T}$.
\end{abstract}

(c) 2014 Elsevier Inc. All rights reserved.

\section{Introduction}

Scalp electroencephalography (EEG) (Niedermeyer and Lopes da Silva, 2005) and functional magnetic resonance imaging (fMRI), typically based on the blood oxygenation level-dependent (BOLD) contrast (Ogawa et al., 1990), are remarkably complementary approaches to study brain function, which has motivated intense efforts towards their combination (Laufs, 2012; Jorge et al., 2013). While EEG and

\footnotetext{
* Corresponding author at: Laboratory for Functional and Metabolic Imaging, LIFMETCIBM, Station 6, École Polytechnique Fédérale de Lausanne, CH-1015 Lausanne, Switzerland.

E-mail address: joao.jorge@epfl.ch (J. Jorge).
}

fMRI can be acquired in separate sessions, and later combined for analysis, simultaneous acquisitions are essential when the features of interest cannot be externally controlled, as in epilepsy (Seeck et al., 1998; Gotman and Pittau, 2011), resting-state activity (Goldman et al., 2002; Britz et al., 2010) and trial-by-trial fluctuations in event-related studies (Scheeringa et al., 2011). Simultaneous acquisitions also avoid differences in spurious stimuli (Novitski et al., 2003), training or habituation effects (Debener et al., 2002) and other differences in subject performance (Boly et al., 2007). Considering fMRI, it is well known that a stronger static field $\mathrm{B}_{0}$ results both in increased magnetization and in an accentuated BOLD effect, leading to super-linear gains in functional sensitivity (Turner et al., 1993; van der Zwaag et al., 2009). As a result, in recent years, fMRI studies conducted at ultra-high field have achieved 
sub-millimeter spatial resolution (Yacoub et al., 2008), and higher field strengths continue to be pursued (Deelchand et al., 2010; Duyn, 2012). These benefits have likewise made simultaneous EEG-fMRI at ultrahigh field an increasingly attractive combination (Neuner et al., 2013).

Unfortunately, simultaneous EEG-fMRI is subject to highly undesirable interactions between the two modalities, which can compromise data quality and raise concerns on subject safety. Safety concerns arise from the possible generation of electric currents along the EEG wires and through biological tissues, induced by the fast-switching MRI gradients or radio-frequency (RF) pulses (Dempsey and Condon, 2001). Additionally, the presence of the conductive EEG materials may alter the transmit $\mathrm{B}_{1}$ field $\left(\mathrm{B}_{1}^{+}\right)$distribution across the head, introducing unpredicted local changes in specific absorption rate (SAR) (Angelone et al., 2004). At 7 T, RF pulse wavelengths become smaller than the typical sample size, greatly increasing the risk of resonant antenna effects along the EEG leads (Dempsey et al., 2001) and creating more heterogeneous $B_{1}$ distributions (Eggenschwiler et al., 2012). The inclusion of current-limiting resistors in the electrodes (Lemieux et al., 1997) and a careful selection of low-SAR MRI sequences (Noth et al., 2012) have been central to minimizing risks of injury. Temperature measurements in phantoms and humans help assessing the magnitude of heating effects (Lazeyras et al., 2001), and electromagnetic (EM) simulations provide high-resolution estimates of the SAR distribution across the head (Angelone et al., 2006).

In addition to safety concerns, simultaneous recordings from both modalities can be affected by severe artifacts, many of which are field strength-dependent. On the one hand, the presence of EEG materials can lead to MR image degradation via two mechanisms: (i) magnetic susceptibility effects between the head tissues and the EEG components, causing localized signal drops and geometric distortions (Krakow et al., 2000), and (ii) $\mathrm{B}_{1}$ disruption or shielding effects caused by the EEG materials, leading to signal-to-noise ratio (SNR) losses. Both mechanisms are field strength-dependent (Mullinger et al., 2008b). With modern EEG configurations, however, even the more conventional silver- or copper-based systems have been found to have an acceptable impact on fMRI data quality at fields up to $3 \mathrm{~T}$ (Bonmassar et al., 2001; Lazeyras et al., 2001). It has further been proposed that temporal SNR in fMRI is relatively well-preserved because physiological noise is also reduced with the overall signal loss (Luo and Glover, 2012).

On the EEG side, MRI-induced artifacts are essentially generated by magnetic induction (Yan et al., 2009). The strongest contributions are usually due to the MRI gradients (Allen et al., 2000), followed by various effects related to the cardiac cycle, altogether known as pulse artifacts (Allen et al., 1998; Mullinger et al., 2013), as well as head rotations in $\mathrm{B}_{0}$, and vibrations propagated from the scanner Helium (He) coldheads (Mullinger et al., 2008a) and ventilation systems (Nierhaus et al., 2013). All these effects scale with $B_{0}$ via their dependence on Faraday's law, with previously less relevant contributions assuming major roles in data degradation at $7 \mathrm{~T}$ (Mullinger et al., 2008a). The pulse artifact has been shown to increase with field strength not only in amplitude but also in spatial variability (Debener et al., 2008). Gradient artifacts depend more directly on slew-rates than on $\mathrm{B}_{0}$ itself, but since spatial resolution is typically higher, slew-rates tend to be pushed as well to quickly achieve the necessary gradient strengths. EEG artifacts can surpass the patterns of interest by several orders of magnitude, and have thus motivated remarkable efforts towards their minimization, both at the level of data acquisition and data analysis (Grouiller et al., 2007; Masterton et al., 2007; Mullinger and Bowtell, 2011).

While EEG noise correction algorithms are currently indispensable, reducing noise contributions during acquisition is undoubtedly the most desirable way to improve data quality, especially at ultra-high field. This can be done, for instance, by reducing the total areas formed by electrode leads between each channel and the reference, thereby reducing magnetic induction effects. In this work, we assessed the importance of EEG cable length and geometry on noise sensitivity, at $7 \mathrm{~T}$, at the level of transmission between the cap and amplifiers. The benefits of shorter signal chains have previously been observed at lower fields (Assecondi et al., 2013), as has the importance of cable geometry (Chowdhury et al., 2012). Here, on a phantom model, the effects of different cable lengths and geometries on EEG recordings were for the first time assessed together, at $7 \mathrm{~T}$, with specific attention given to $\mathrm{He}$ coldhead contributions (Study I). An optimized EEG setup with ultrashort bundled cables (approximately $12 \mathrm{~cm}$ from cap to amplifiers) was implemented (Fig. 1), and a series of safety tests were conducted, including EM simulations on a realistic head model and surface temperature measurements on a phantom during SAR-intensive fMRI acquisition. The effects of the optimized setup on MRI data quality were also extensively assessed in one volunteer (Study II). Finally, this setup was employed for simultaneous EEG-fMRI acquisition on 5 healthy volunteers under two visual paradigms: an eyes-open/eyes-closed task and a visual evoked potential (VEP) run using reversing-checkerboard stimulation (Study III).

\section{Methods}

\section{MRI system}

All measurements reported in this work were performed on an actively-shielded Magnetom 7 T head-only scanner (Siemens, Erlangen, Germany), with ultra-short bore length (Magnex Scientific, Oxford, UK) and $680 \mathrm{~mm}$ bore diameter (Fig. 1a). The scanner was equipped with an AC84 head gradient set (max. slew-rate $333 \mathrm{~T} / \mathrm{m} / \mathrm{s}$ ) and a custom-built 8-channel transmit/receive loop head array (Rapid Biomedical, Rimpar, Germany; Fig. 1c).

\section{Optimized EEG setup}

For study I, EEG data were acquired as described in the corresponding EEG acquisition section. For study II and study III, an optimized acquisition setup was used: EEG data were recorded using two 32channel BrainAmp MR Plus amplifiers (Brain Products, Munich, Germany) and a customized BrainCap MR model (EasyCap, Herrsching, Germany). The cap contained $64 \mathrm{Ag} / \mathrm{AgCl}$ ring-type electrodes ("multitrodes"), arranged according to the international 10-20 system, and was designed with shortened electrode leads terminating in two connectors at approximately $1-3 \mathrm{~cm}$ from the cap surface (Fig. 1b). Each copper electrode lead contained a $5 \mathrm{k} \Omega$ resistor near the electrode and another inside the connector. One of the 64 electrodes was placed on the back of the subject for electrocardiogram (ECG) recording. The cap connectors were linked to the EEG amplifiers via two $12 \mathrm{~cm}$ bundled cables (Fig. 1d). The two amplifiers rested on top of each other just outside the head array, on the RF gateway box (Fig. 1a). After bandpass filtering $(0.1-250 \mathrm{~Hz})$ and digitization $(0.5 \mu \mathrm{V}$ resolution), the EEG signals were transmitted to the control room via two fiber optic cables. EEG sampling was performed at $5 \mathrm{kHz}$, synchronized with the scanner $10 \mathrm{MHz}$ clock. In both phantom and human recordings, Abralyte gel (EasyCap) was used to reduce electrode impedances. The scanner He coldheads were kept in function at all times in both studies.

\section{Study I: EEG cable noise contributions}

This study assessed EEG noise sensitivity depending on the length and geometry of the ribbon cables connecting the cap to the amplifiers. EEG recordings were performed on an agar gel phantom, with no concurrent MRI acquisition. Patient ventilation, room and bore lights, and the scanner host remained switched off at all times. Recordings were performed both with and without the scanner coldheads in function.

\section{EEG acquisition}

In this study, EEG data were recorded using a single 32-channel BrainAmp MR Plus amplifier connected via a ribbon cable to an MRcompatible signal tester box. This signal tester, where each channel is 
a)

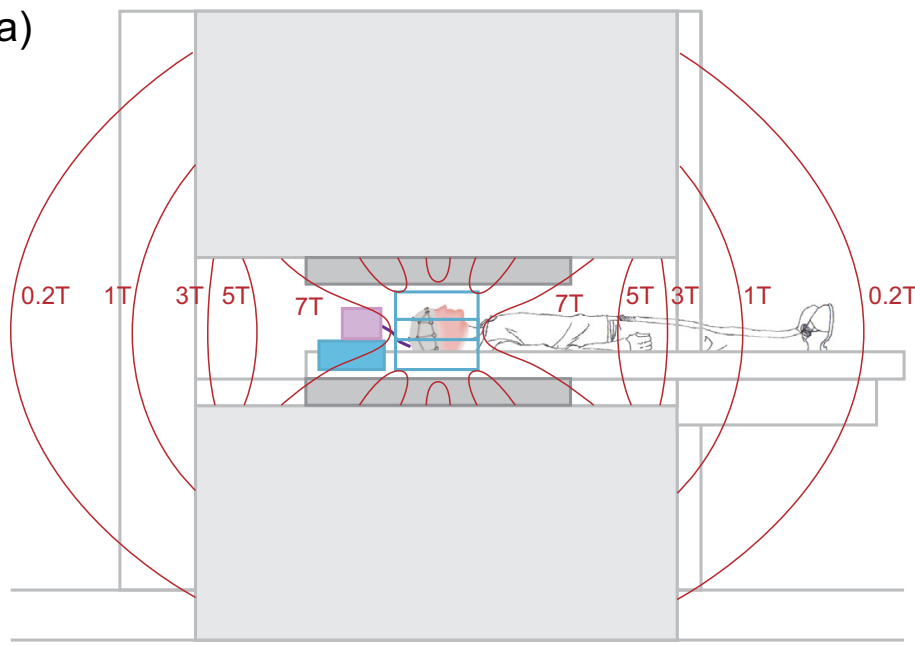

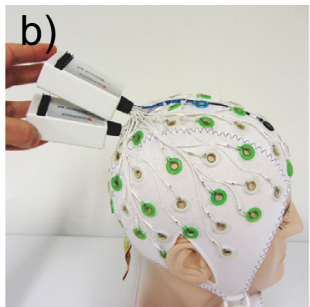

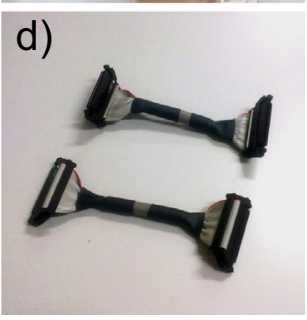

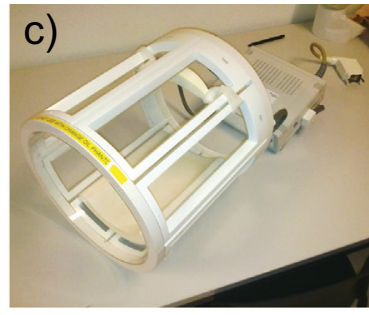

e)

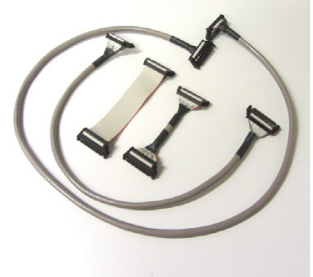

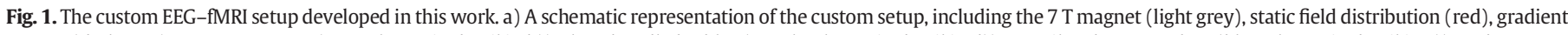

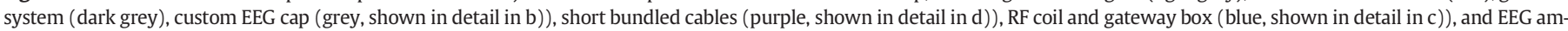

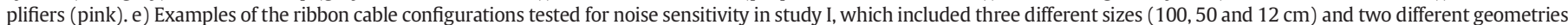
(flat and bundled). All components are shown with permission from the respective manufacturers.

directly linked to the reference via a $5 \mathrm{k} \Omega$ resistor, was then tightly fixed to the top (head side) of the phantom. This approach avoided the use of an actual EEG cap, so as to capture strictly cable-related noise contributions. EEG signals were recorded for approximately 2 min for each configuration, with a $500 \mathrm{~Hz}$ sampling frequency, a hardware bandpass filter of $0.1-250 \mathrm{~Hz}$, and $0.5 \mu \mathrm{V}$ amplitude resolution.

\section{Cable configurations}

A total of 6 different ribbon cables were tested, comprising 3 different lengths (100, 50, and $12 \mathrm{~cm}$ ) and 2 different geometries: (i) the typical flat ribbon configuration, with the reference channel running approximately in the middle, and (ii) a bundled configuration where all channels are tightly bunched together in a cylindrical shape (Figs. 1d-e). For the shortest cable length $(12 \mathrm{~cm})$, the EEG amplifier was placed on top of the RF coil gateway box; for longer cable lengths, the amplifier was suspended on a wooden support mechanically isolated from the bed, maintaining a similar position relative to the central axis of the scanner bore.

\section{EEG data analysis}

Following acquisitions, EEG noise contamination was assessed and compared across different cable configurations. A qualitative comparison relied on visual inspection of channel timecourses and channel-averaged noise spectra, estimated via fast Fourier transform over a $30 \mathrm{~s}$ period. For a quantitative comparison between configurations, an average full-spectrum noise power estimate was computed for each channel in each condition, based on the mean of the squared signal over $30 \mathrm{~s}$. These estimates were then statistically analyzed via a 3-way ANOVA, incorporating the factors of cable length, cable geometry, and He coldhead state.

\section{Study II: safety and MRI data quality}

A series of tests were performed to evaluate the impact of the optimized setup (12 cm bundled configuration) on subject safety and EEG amplifier integrity, as well as its degrading effects on MR image quality. During acquisitions, the patient ventilation system, room and bore lights remained switched off at all times.

\section{Electromagnetic simulations}

EM simulations were performed to evaluate the impact of the custom EEG setup on $\mathrm{B}_{1}^{+}$and SAR distributions across the head, as generated by the RF loop volume array used in this work. The measurement setup was simulated with the finite difference time domain (FDTD) package SEMCAD X (SPEAG, Zürich, Switzerland), using the realistic human meshed model Duke from the Virtual Family (Christ et al., 2010). For the RF coil, the copper strips of the loop array were modeled as perfect electric conductors (PEC), with capacitors and voltage sources inserted on each loop to ensure excitation of the circularly polarized mode. EEG ring electrodes were designed as a set of 63 PEC loops, connected to PEC leads via $5 \mathrm{k} \Omega$ resistors. The leads converged in 8 branches towards the 2 connectors, standing approximately $2 \mathrm{~cm}$ above the scalp (Fig. 2a). Wire branching and connector positions were modeled according to the real cap (Fig. 1b), with specific care to ensure that no wires/electrodes were in physical contact with each other or the skin. Contact with the scalp was modeled with small cylinders mimicking the Abralyte gel (Fig. 2b), with dielectric properties measured from a real gel sample using a dielectric probe (SPEAG, Zürich, Switzerland). The simulation model was meshed in a non-uniform grid of approximately 8MCells, with voxeling steps ranging from $0.26 \times 0.40 \times 0.29 \mathrm{~mm}^{3}$ to $69 \times 78 \times 85 \mathrm{~mm}^{3}$ (Fig. 2b). A harmonic excitation at $297.2 \mathrm{MHz}$ was applied, and steady-state conditions were achieved within 30 periods of simulation time. Perfectly matched layers in medium strength were used at the edges of the FDTD domain. The resulting $\mathrm{B}_{1}^{+}$and SAR maps, with and without the cap, were normalized to a $1 \mathrm{~W}$ delivered power and exported to Matlab (Mathworks, Natick MA, USA) to be resampled into a uniform grid.

\section{Temperature measurements}

Temperature monitoring was conducted on an agar gel phantom contained in a realistic head shape, which was covered with an Abralyte gel layer and fitted with the EEG cap. Measurements were performed using a 4-channel fiber optic temperature sensor (Neoptix, Québec, Canada), with two probes placed on electrodes AF8 and FT9 (directly in the gel within the ring electrodes), one probe in between the two EEG amplifiers, and another suspended from the head coil, above the phantom, for reference. Temperature fluctuations were assessed during a 16 min session where two fMRI runs were applied sequentially, for 8 min each: a sinusoidal gradient-echo (GE) EPI sequence (25 axial slices, $1.5 \times 1.5 \times 1.5 \mathrm{~mm}^{3}$ spatial resolution with $1.5 \mathrm{~mm}$ interslice gaps, TR/TE $=2000 / 25 \mathrm{~ms}, \alpha=78^{\circ}, 69 \%$ of SAR limit), followed by a spin-echo (SE) EPI sequence (20 slices, $1.5 \times 1.5 \times 1.5 \mathrm{~mm}^{3}$ resolution, $\mathrm{TR} / \mathrm{TE}=5000 / 44 \mathrm{~ms}, \alpha=90^{\circ}, 91 \%$ of SAR limit). The two runs were separated by approximately 2 min, which included shimming and 
a)

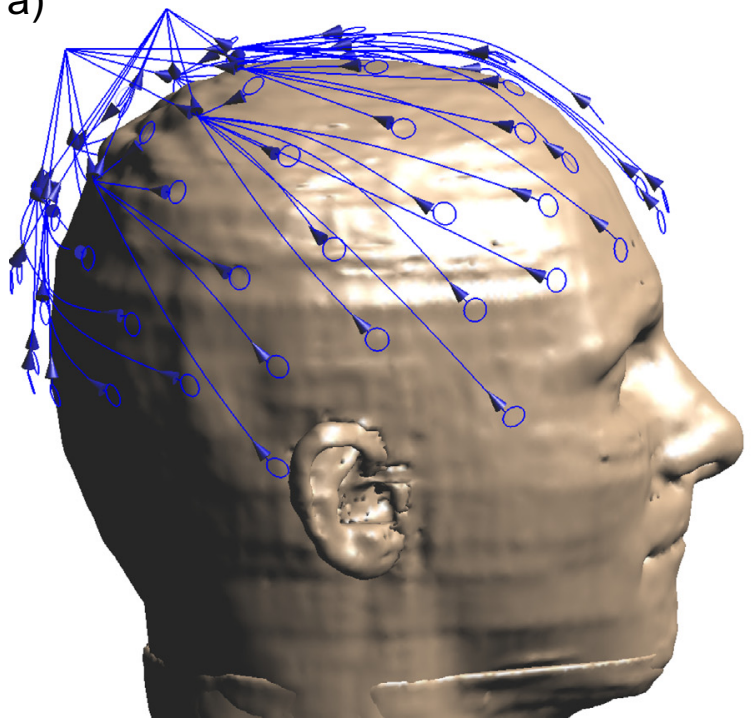

b)

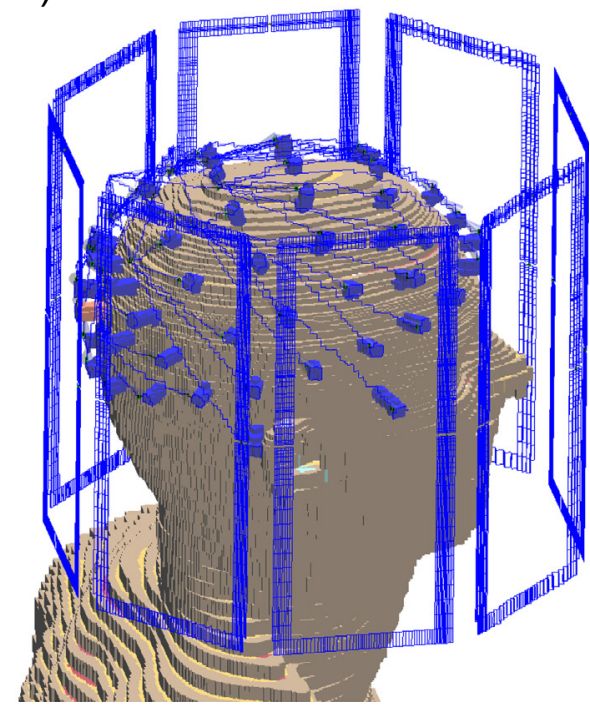

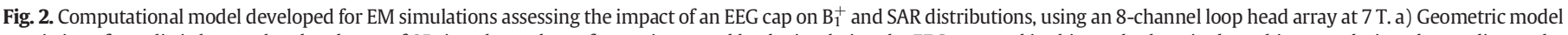

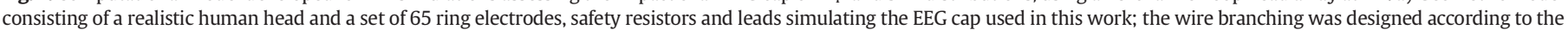

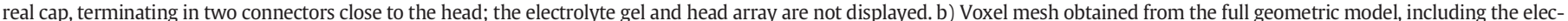
trolyte gel and head array.

other adjustment procedures for the second run. No MRI acquisitions had been performed on the scanner for several hours prior to this session.

\section{MRI data quality}

In order to evaluate MR image degradation effects, one human volunteer was scanned with and without the EEG setup in place. For both cases, a set of four sequences was applied: a GE-EPI volume (same parameters as in the Temperature measurements) to assess fMRI image quality, a GRE anatomical image (176 sagittal slices, $1.0 \times 1.0 \times 1.0 \mathrm{~mm}^{3}$ spatial resolution, TR/TE $=6.5 / 2.8 \mathrm{~ms}, \alpha=4^{\circ}$ ), a GRE-based $\mathrm{B}_{0}$ field map (30 slices, $3.0 \times 3.0 \times 3.0 \mathrm{~mm}^{3}$ resolution, $\mathrm{TR} / \mathrm{TE}_{1} / \mathrm{TE}_{2}=1050 / 4 / 5 \mathrm{~ms}$, $\alpha=40^{\circ}$ ), and a SA2RAGE image (64 sagittal slices, $2.0 \times 2.5 \times 2.0 \mathrm{~mm}^{3}$ resolution, TR/TE $=2400 / 1.4 \mathrm{~ms}, \mathrm{TI}_{1} / \mathrm{TI}_{2}=65 / 1800 \mathrm{~ms}, \alpha_{1} / \alpha_{2}=$ $4^{\circ} / 11^{\circ}$ ) for $\mathrm{B}_{1}^{+}$field mapping (Eggenschwiler et al., 2012). When present, the EEG setup was kept operating similarly to a normal recording.

Data quality assessment and comparison were performed through direct visual inspection, and additionally, for functional and anatomical images, through the estimation of SNR losses. Spatial SNR was estimated as the mean amplitude in a specific region within the head divided by the standard deviation of signal amplitudes outside the head (avoiding areas potentially affected by Nyquist ghosting). Two head regions were considered: one comprising more inferior areas not visibly affected by accentuated signal drops, for a general measure of SNR loss, and another comprising more superior regions affected by pronounced signal losses. Given the importance of temporal, rather than spatial SNR for fMRI (Luo and Glover, 2012), temporal SNR values were obtained from the fMRI data acquired during VEP runs, with concurrent EEG acquisition (described in detail in Study III: simultaneous acquisitions in humans), and compared to values from an fMRI-only dataset. Due to time constraints, this second dataset was acquired from a different subject group ( $\mathrm{N}=4$, similar age range), using the same MRI setup and sequence parameters. For each subject run, temporal SNR was calculated for each voxel as the mean timecourse amplitude divided by the timecourse standard deviation, and then averaged within a frontal white matter region $(7 \times 7 \times 7$ voxels $)$ not affected by accentuated signal loss. All estimated spatial and temporal SNR values were above 10 , and therefore a compensation for the Rician distribution of the noise was not necessary (Triantafyllou et al., 2005). The effect of GRAPPA acceleration on background noise was present, but similar on both conditions.

\section{Study III: simultaneous acquisitions in humans}

Human tests comprised the final stage of this work, intended to assess BOLD and EEG data quality using the optimized setup, particularly in terms of functional sensitivity and potential use for single-trial studies. Five healthy male volunteers ( $20 \pm 2$ years old) participated in this study, having provided written informed consent. The study had been previously approved by the institutional review board of the local ethics committee. Due to time constraints, one of the volunteers did not undergo the eyes-open/eyes-closed run.

\section{Functional paradigms}

Volunteers underwent two functional runs: (i) an eyes-open/eyesclosed run mediated by auditory cues, and (ii) a VEP run applying reduced-field reversing-checkerboard stimuli. The eyes-open/closed run comprised eight blocks of $15 \mathrm{~s}$ eyes-closed followed by $15 \mathrm{~s}$ eyesopen periods. Instructions were given to the subjects from the control room via the patient communication system, with the room lights kept ON throughout the experiment. For the VEP run, checkerboards were presented during eight 10s blocks at a reversal frequency of $4 \mathrm{~Hz}$ (totaling 39 reversals per block), followed by 20s of rest (fixation). A red cross was shown at the center of the field of view (FOV) at all times, with slight shifts in color occurring twice per block at random time delays. Subjects were instructed to remain focused on the cross and report color shifts via a button press. Checkerboards were presented at $50 \%$ contrast, maintaining an equivalent average luminance to the rest periods. The stimulation FOV was limited to approximately $7^{\circ}$, a fairly selective central-field stimulus (ACNS, 2006). This was both due to technical limitations (the images were projected from the back of the bore, with the EEG amplifiers partially obstructing the FOV), and intentionally in order to assess sensitivity for a weaker stimulus, especially on a single-trial scale. As the stimulation was performed using an LCD projector, a StimTracker box (Cedrus Corporation, San Pedro CA, USA) equipped with a photodiode sensor was used to record the precise timing of checkerboard reversals. Room lights were kept OFF for this run. The two 4-minute runs were presented in counter-balanced order 
across subjects, separated by a short pause for communication with the subject and pre-scanning adjustments.

\section{EEG-fMRI acquisition}

Simultaneous acquisitions were performed with the optimized EEG setup described in the Optimized EEG setup section, and the GE-EPI sequence described in the Temperature measurements section, this time with only $5 \%$ interslice gaps. The EPI volume slab was placed in an axial-oblique orientation fit to contain as much of the primary visual cortex as possible. Volume triggers were received from the scanner at the start of each EPI volume and recorded along with the EEG traces. Bore lights and the patient ventilation system were kept OFF throughout the sessions, with no discomfort reported by the subjects.

\section{EEG data analysis}

Data analysis was performed in Matlab using routines developed in-house. For each run, based on the recorded volume triggers, slice triggers were produced by splitting each volume interval in 25 equal segments. The resulting slice triggers were then fine-tuned by maximizing inter-slice correlations, in a $10 \times$-upsampled EEG channel (Niazy et al., 2005). Gradient artifacts were then corrected slice-by-slice via average artifact subtraction (AAS) and optimal basis set (OBS) techniques (Allen et al., 2000; Niazy et al., 2005). For AAS, each slice was subtracted of an average over 50 slice samples ( 25 from the preceding and 25 from the following slices), with randomly-jittered steps of 4-6 slices separating the selected samples. With this spacing, the samples selected for averaging were thus spread over a period of approximately $20 \mathrm{~s}$ (250 slices) centered on the slice to be corrected. Step jittering was applied to mitigate the removal of EEG activity of interest, especially for VEP data, where stimulation ( $250 \mathrm{~ms}$ period) was phase-locked with slice acquisition ( $80 \mathrm{~ms}$ ) every 25 slices. Furthermore, the LCD projector used for checkerboard presentation had a variable delay of $0-17 \mathrm{~ms}$ (which was monitored with a photodiode for VEP triggering), providing an additional jitter to the acquisition-stimulation timing relationship. Cardiac triggers were estimated from the ECG channel and fine-tuned by another correlation-maximization approach, using a combination of EEG channels where pulse artifacts were most prominent. Based on these triggers, pulse artifacts were reduced via OBS, using the 3-5 most important principal components, depending on the stability of this artifact throughout each run. Data were then downsampled to $500 \mathrm{~Hz}$, and bad channels were identified (1-5 per dataset) and replaced by weighted averages of 3-4 neighboring electrodes. For the eyes-open/closed run, data were re-referenced to the average reference, decomposed via independent component analysis (ICA), and then reconstructed by manual selection of the components exhibiting differences in alpha power $(8-12 \mathrm{~Hz})$ between eyes-open and eyes-closed conditions. For the VEP run, data were bandpass filtered to $4-30 \mathrm{~Hz}$, re-referenced to the average reference, and decomposed via ICA. The datasets were then reconstructed by manual selection of components displaying non-artifactual, potentially VEP-related dynamics, based on their topography, trial average response and trial-by-trial variability (Arrubla et al., 2013; Neuner et al., 2014). Component selection was always performed by the same operator, using similar criteria.

\section{fMRI data analysis}

Data analysis was performed using custom routines implemented in Matlab. For both runs, fMRI data underwent motion correction, slicetiming adjustments, brain segmentation, spatial smoothing $(2 \mathrm{~mm}$ FWHM) and temporal de-trending (Smith et al., 2004). The datasets were then analyzed voxel by voxel with a general linear model (GLM) approach (Worsley and Friston, 1995). Here, a "boxcar" timecourse was designed for each experiment (1's during eyes-closed periods and 0 's during eyes-open periods, for the eyes-open/closed run, and 1 's during checkerboard stimulation and 0's during fixation, for the VEP run). These binary timecourses were then convolved with a (double- gamma) canonical hemodynamic response function. Motion parameters were also included in all models as confounds.

\section{Results}

\section{Study I: EEG cable noise contributions}

Based on preliminary tests, the scanner electronic hardware, room and bore lights, and host computer were found to have a negligible effect on EEG signal quality. The patient ventilation system produced relevant noise contributions at frequencies below $30 \mathrm{~Hz}$, but could be switched off throughout all recordings without relevant consequences. With the scanner coldheads in function, using a $100 \mathrm{~cm}$ conventional (flat) ribbon cable, most EEG channels clearly displayed a stationary noise pattern of high frequency oscillations, with a fundamental period of approximately $1 \mathrm{~s}$ (Fig. 3, left). This pattern disappeared upon switching off the coldheads. In the setup used for this study, channel numbers were attributed in sequence according to the position of each wire lane running along the cable, with the reference channel running approximately in the middle (between channels 16 and 17, and most distant from channels 1 and 32). A progressive increase in noise amplitude was clearly seen for channels running farther away from the reference, as would be expected from an artifact generated by magnetic induction. This trend was quantified by computing full-spectrum noise power estimates for each channel and then comparing these values with results obtained with a similar configuration but using a bundled cable (Fig. 3, right). The dependence of channel noise power on the distance to the reference was evident for the flat type, but became greatly attenuated in the bundled configuration. Over all channels, for this cable length of $100 \mathrm{~cm}$, the bundled type yielded a reduction of $58 \%$ in channel-averaged total noise power, and an $81 \%$ reduction in inter-channel noise power variability.

To compare the different cables tested, channel-averaged noise spectra were computed for each configuration. Different cable lengths and geometries displayed distinct overall power amplitudes, along with some differences in spectral distribution (Supplementary Fig. 1). The scanner coldheads showed a major impact on noise amplitudes in the range of 20 up to $150 \mathrm{~Hz}$, producing a considerable fraction of total noise power. The source(s) of the remaining noise could not be experimentally identified, but were confirmed not to be caused by the patient ventilation system, room or bore lights, scanner hardware or the host computer.

For a quantitative comparison between cable configurations, a fullspectrum noise power estimate was computed for each channel in each condition. Channel-averaged results are presented in Fig. 4. The influence of cable length and geometry on noise power was found highly statistically significant, as was the impact of coldhead contributions (all 3 effects with $\mathrm{p}<0.01$ ). Over all tested lengths, bundled cables yielded reductions of $0.2-69 \%$ in total noise power relative to flat cables, with the coldheads switched OFF, and of $43-63 \%$ with the coldheads ON. Inter-channel variability was reduced by $18-88 \%$ with the coldheads OFF and by $47-81 \%$ with the coldheads ON when using bundled cables. Conversely, over the two geometry types, shortening from 100 to $12 \mathrm{~cm}$ yielded reductions of $44-70 \%$ in total noise power with the coldheads OFF and of $58-62 \%$ with the coldheads ON. Inter-channel variability was reduced by $59-83 \%$ with the coldheads OFF and by $52-63 \%$ with the coldheads $\mathrm{ON}$, through cable shortening. Overall, the combination of cable bundling and shortening (from 100 to $12 \mathrm{~cm}$ ) led to a reduction of $84 \%$ in total noise power and of $91 \%$ in inter-channel noise power variability, with the coldheads in function.

\section{Study II: safety and MRI data quality}

\section{Electromagnetic simulations}

To assess the impact of the custom EEG cap on $\mathrm{B}_{1}^{+}$and SAR distributions across the head, EM simulations performed with and without the 

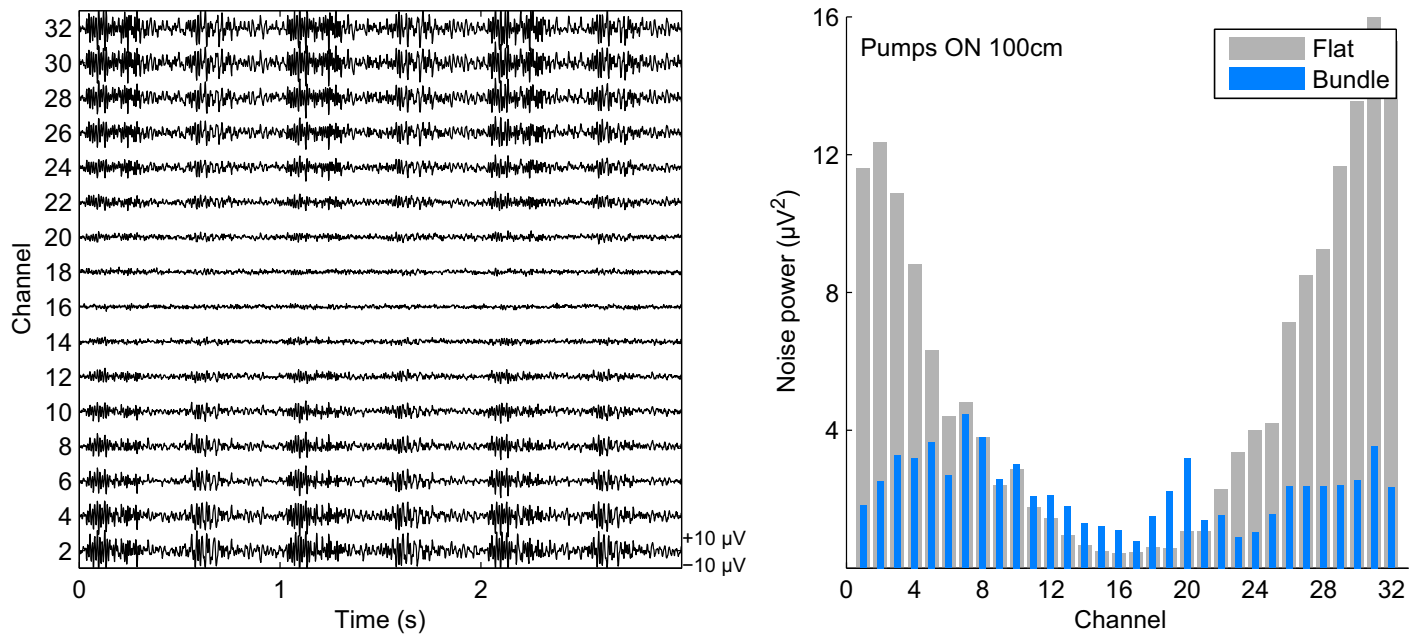

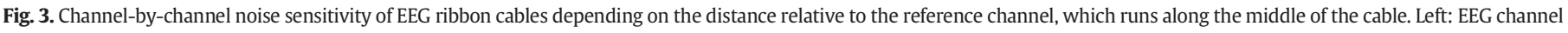

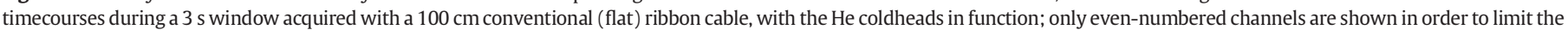
figure size. Right: average noise power in each channel over a $30 \mathrm{~s}$ period, for both flat and bundled ribbon cables, $100 \mathrm{~cm}$ long, with the coldheads in function.

cap in place were compared (Fig. 5). The presence of the EEG materials led to a general loss in $\mathrm{B}_{1}^{+}$amplitude-approximately 8.0\% over the head region. The general properties of the field distribution, with higher amplitude in the center and in occipital regions, were roughly maintained with the inclusion of the EEG cap. Nevertheless, a number of local, more accentuated effects were observed in superior regions, mostly restricted to the scalp, especially in the vicinity of EEG leads (Fig. 5, arrow 1 ). These local effects included both field decreases and increases, in some voxels up to $1.7 \times$ the nominal flip angle. SAR maps (averaged over $10 \mathrm{~g}$ of tissue) expressed similar trends, with the introduction of the EEG cap leading to an overall decrease of approximately $7.9 \%$ over the whole head. A few local increases could be observed in superior-anterior regions, close to the skin (Fig. 5, arrow 2), pushing the peak $10 \mathrm{~g}$ average SAR value from $0.39 \mathrm{~W} / \mathrm{Kg}$ without EEG to $0.43 \mathrm{~W} / \mathrm{Kg}$ with the cap (normalized to $1 \mathrm{~W}$ power).

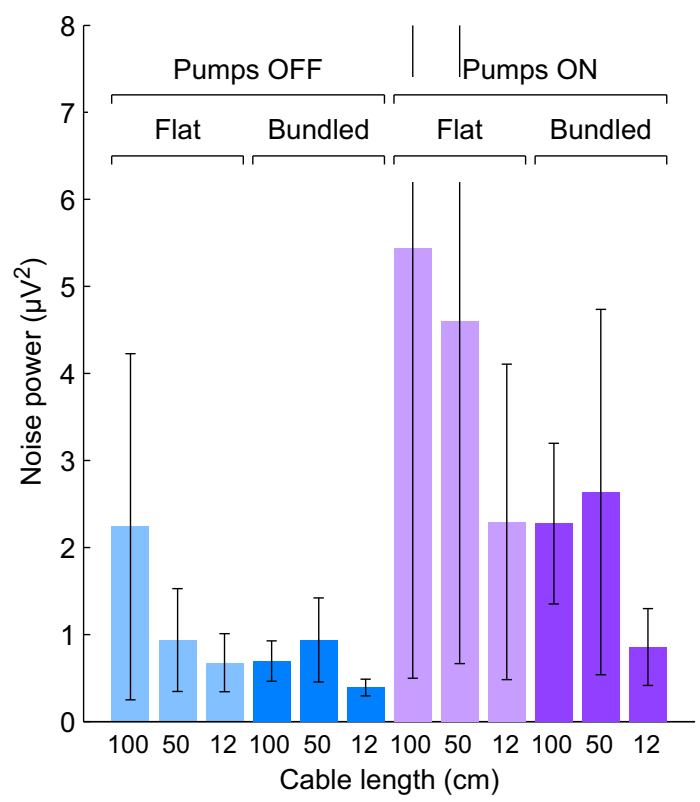

Fig. 4. Average EEG noise power for different ribbon cable configurations, with the He coldheads turned on as well as off. For each channel, noise power estimates were obtained from 30 s periods. Bar heights represent channel averages, and error bars represent the standard deviation across channels.
The validity of the EM simulations was assessed by comparing the estimated $\mathrm{B}_{1}^{+}$maps with real measurements performed on a human subject, with and without the EEG cap in place, as described in the MRI data quality section (Fig. 6). In general, the in vivo measurements exhibited similar field distributions to the simulated maps, with higher $\mathrm{B}_{1}^{+}$in the center and occipital regions, and a $12.8 \%$ overall decrease in $\mathrm{B}_{1}^{+}$ strength upon introduction of the EEG cap. Local $\mathrm{B}_{1}^{+}$deviations occurring closer to the skin effectively differed in location and shape, but expressed similar intensity variations, with in vivo measurements showing decreases down to near-complete $\mathrm{B}_{1}^{+}$loss and increases up to approximately $1.8 \times$ the nominal flip angle.

\section{Temperature measurements}

Complementary to EM simulations, local heating effects due to the EEG system were assessed by temperature monitoring on a phantom during a GE-EPI followed by a spin-echo EPI acquisition. During both 8 min runs, no significant temperature increases were found in any of the monitored locations apart from the EEG amplifiers (Supplementary Fig. 2). In the reference probe suspended above the phantom, temperature increased from 19.7 to $20.0{ }^{\circ} \mathrm{C}$ in the GE run and then up to $20.3{ }^{\circ} \mathrm{C}$ during the SE run. The 2 probes placed on EEG electrode sites exhibited similar trends, at slightly lower temperatures, with total increases below $1{ }^{\circ} \mathrm{C}-$ on AF8, temperature rose from 18.2 to $18.7{ }^{\circ} \mathrm{C}$ (GE) and then up to $19.1{ }^{\circ} \mathrm{C}$ (SE); FT9 showed an increase from 17.8 to $18.1{ }^{\circ} \mathrm{C}$ (GE) and then up to $18.4{ }^{\circ} \mathrm{C}(\mathrm{SE})$. The sensor placed on the EEG amplifiers did measure stronger heating effects: from 21.4 to $25.8{ }^{\circ} \mathrm{C}$ in the GE run, and then up to $27.9{ }^{\circ} \mathrm{C}$ during the SE run.

\section{MRI data quality}

The impact of the EEG system on MRI data quality for a human subject was assessed in functional and anatomical images acquired with and without the EEG system, and its underlying mechanisms were investigated via $\mathrm{B}_{0}$ and $\mathrm{B}_{1}^{+}$mapping (Fig. 6). Both functional (GE-EPI) and anatomical (GRE) images exhibited general losses in spatial SNR of approximately $37 \%$ and $29 \%$, respectively. Central-superior regions were particularly affected (arrows 1-2 in Fig. 6), with SNR losses rising to $62 \%$ (functional) and $44 \%$ (anatomical) in the top-most axial slices. In the fMRI datasets, on average across subjects, white matter temporal SNR values decreased from $22 \pm 1$ in fMRI-only runs to $17 \pm 1$ in EEG-fMRI runs, corresponding to an average loss of $23 \pm 6 \%$.

Regarding field mapping results, with the EEG system in place, the $B_{0}$ distribution did evince a number of local inhomogeneities along the scalp (arrow 3 in Fig. 6), likely corresponding to individual electrodes 

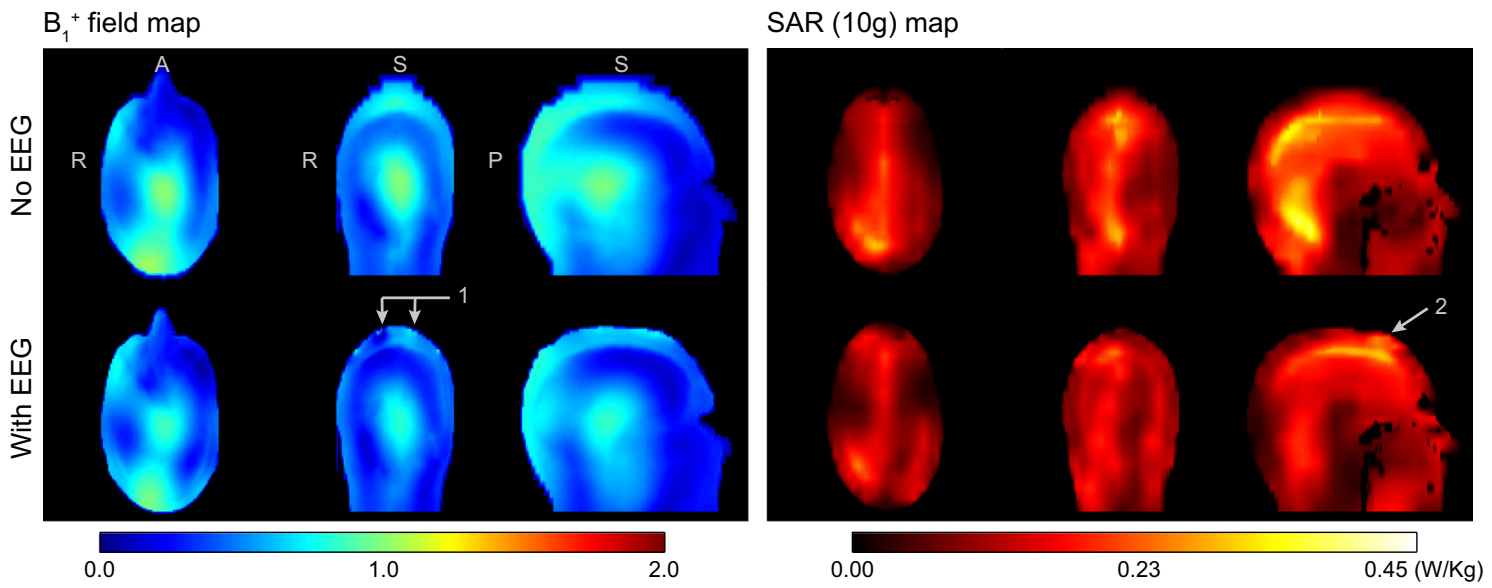

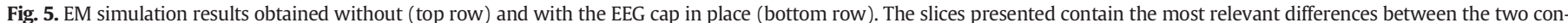

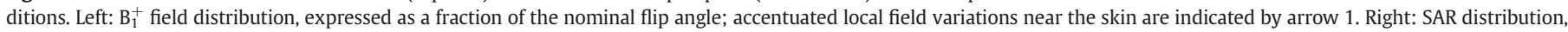
expressed as the average value over $10 \mathrm{~g}$ of tissue; a moderate increase in SAR in superior-anterior regions, near the scalp, is indicated by arrow 2.

and gel. However, these inhomogeneities were in general limited to extra-cerebral tissue, whereas in the brain the $\mathrm{B}_{0}$ distribution remained comparable to the no-EEG situation. This is in agreement with the observation that no geometric deformations were found in GE-EPI or GRE images with the introduction of the EEG cap. In contrast, $\mathrm{B}_{1}^{+}$maps evinced clear differences between the two conditions, including both a general $12.8 \%$ loss in amplitude over the whole head and a number of more accentuated local effects (arrow 4 in Fig. 6), as predicted by the
EM simulations (Electromagnetic simulations section). Relevant increases were mainly located outside the brain, while accentuated local decreases could also be seen in deeper regions.

\section{Study III: simultaneous acquisitions in humans}

The feasibility of simultaneous EEG-fMRI at $7 \mathrm{~T}$ using the proposed setup was evaluated in 5 healthy volunteers, none of which reported
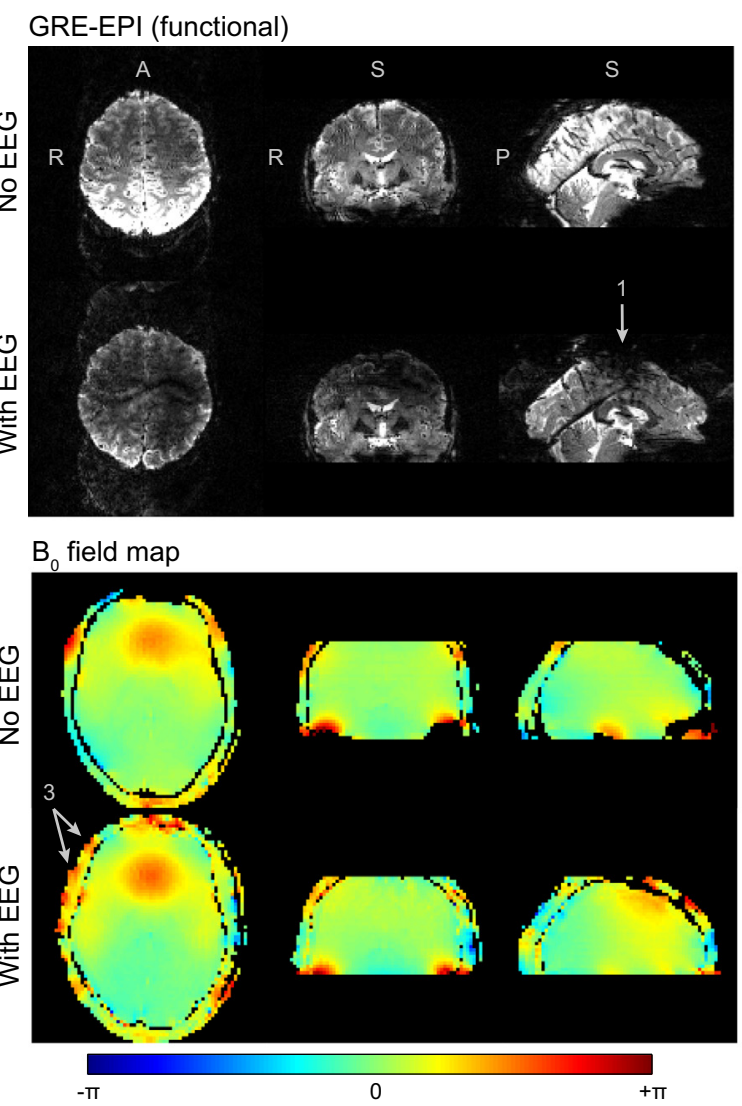

GRE (anatomical)

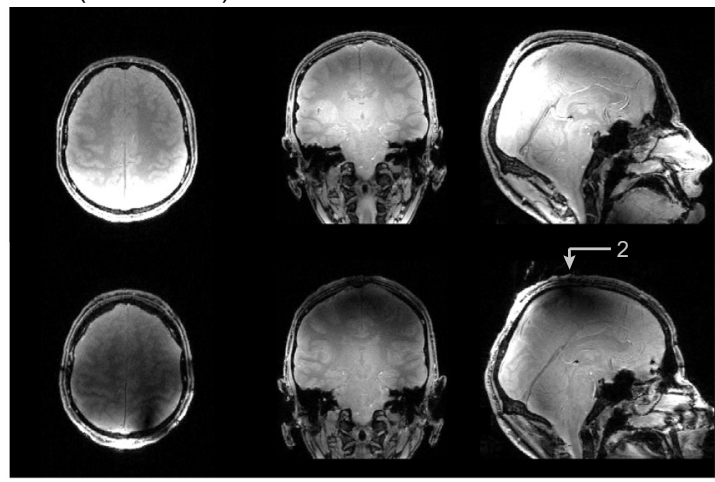

$\mathrm{B}_{1}^{+}$field map

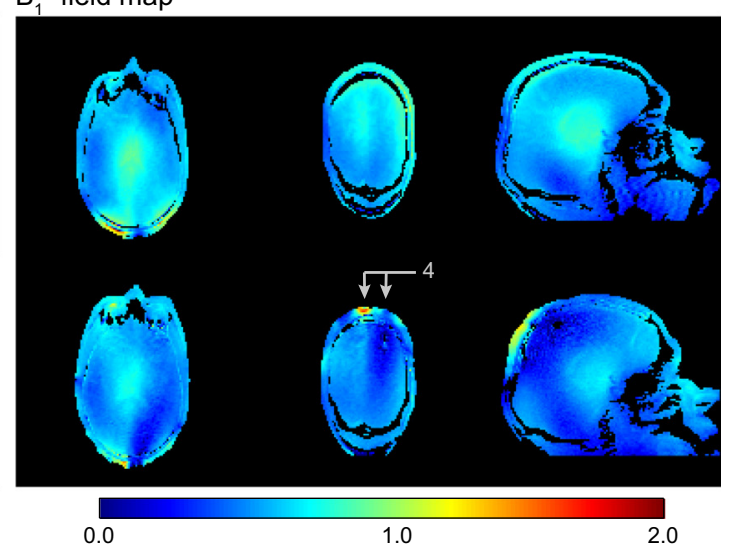

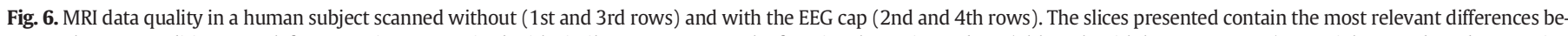

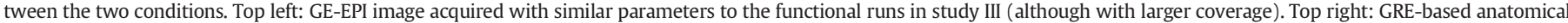

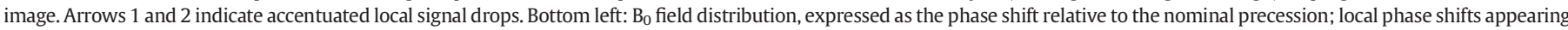

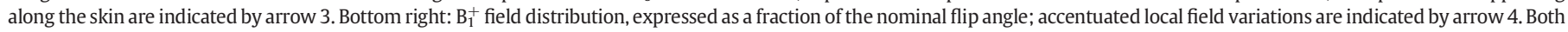
$\mathrm{B}_{0}$ and $\mathrm{B}_{1}^{+}$field maps here shown were masked to remove background regions in which there was no MR signal. 
any unusual skin heating effects. Likewise, the EEG amplifiers operated normally throughout all runs, without heating-related impairments.

\section{EEG data}

Gradient and pulse artifact correction steps produced strong changes on the original data (Supplementary Fig. 3). Across all subjects and paradigms, full-spectrum (1-250 Hz) EEG power was reduced by $99.6 \pm 0.1 \%$ with AAS-based gradient artifact correction, subsequently by $1.9 \pm 0.4 \%$ with OBS-based residual gradient artifact correction, and finally by $64.3 \pm 5.9 \%$ with OBS-based pulse artifact correction (each reduction estimated relative to the data from the preceding correction step). Following ICA decomposition, 5-7 sources per subject were selected as relevant from the eyes-open/closed data, and 4-6 sources were selected from the VEP data (Supplementary Figs. 4 and 5). The ICA-reconstructed EEG data from the eyes-open/closed run revealed accentuated alpha modulation in occipital channels (Fig. 7a). Alpha power increases could be clearly observed in single-channel timecourses during most of the eyes-closed blocks, compared to eyesopen periods (Fig. 7b). The 4th subject did not show any task-related alpha power variations, consistent with the absence of any significant task-related BOLD signal changes (data not shown).

For the VEP run, all 5 subjects exhibited an average response in occipital regions dominated by a positive peak occurring approximately $100 \mathrm{~ms}$ after stimulus onset (checkerboard reversal), commonly known as the P100 component (Bonmassar et al., 1999; Mahajan and McArthur, 2012). Over the scalp, the P100 peak reflected an anteriorposterior dipole (Fig. 8a), dominating the average global field power (GFP) response at the same latency (Fig. 8b), in good agreement with previous reports (Skrandies, 2005). On a single-trial scale, occipital responses were considerably noisier, with only a moderate fraction of the trials exhibiting a clear response pattern consistent with the average VEP (Fig. 8c). Nevertheless, a trial-by-trial regression analysis using a 2-regressor model, comprising the average VEP and its temporal derivative (to allow for variability in visual response latencies), showed that statistically significant responses $(p<0.05)$ were found in 164-177 trials out of 312 for this group of 5 subjects.

\section{fMRI data}

In the eyes-open/closed run, statistically significant negative BOLD signal changes were detected for eyes-closed periods in occipital regions in the same 3 out of 4 subjects showing significant EEG responses (Fig. 9a). Across these subjects, average Z-scores within significantly active regions $(Z<-3.5)$ ranged from -5.6 to -4.4 , with percent signal changes of $-3.9 \%$ to $-3.7 \%$. Peak Z-scores ranged from -11.1 to -9.4 . For the VEP run (Fig. 9b), statistically significant positive signal changes, correlated with checkerboard stimulation periods, were detected in occipital regions for all 5 subjects. Average Z-scores within significantly active regions $(Z>+3.5)$ ranged from +4.9 to +5.2 , with percent signal changes of $+3.0 \%$ to $+3.8 \%$. Peak $Z$-scores ranged from +10.4 to +13.5 .

\section{Discussion}

The present work demonstrates clear benefits in EEG cable shortening and bundling for artifact prevention, at the level of signal transmission between collection (EEG cap) and amplification (after which the signals are digitized). An optimized setup with ultra-short bundled transmission cables was implemented and tested for simultaneous EEG-fMRI, at $7 \mathrm{~T}$. After assessing the impact of this setup on safety and MRI data quality, simultaneous acquisitions were performed in a group of 5 volunteers undergoing two visual paradigms, and the resulting data were analyzed to assess trial-average and single-trial response detection sensitivity.

\section{EEG setup optimization}

The noise measurements performed in study I provide clear insights into the importance of EEG setup optimization. Consistent with previous studies (Mullinger et al., 2008a), the He coldheads were shown to have a major impact on EEG recordings performed at $7 \mathrm{~T}$, producing the largest noise contributions in the absence of gradient, pulse, and subject motion artifacts. Spanning a wide range of frequencies, well within the relevant EEG domain, coldhead-related contributions exhibited complex spectral profiles that are likely to depend on a multitude of properties of the overall mechanical system linking the coldheads, EEG amplifiers, patient bed and the patient itself. While these contributions can be fully avoided by switching off the compression systems during acquisition (Mullinger et al., 2008a; Ritter et al., 2010), this procedure is simply not allowed in many clinical and research sites, and becomes a)

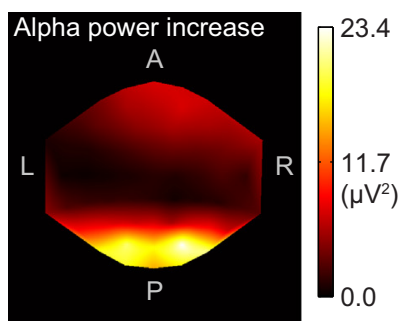

b)

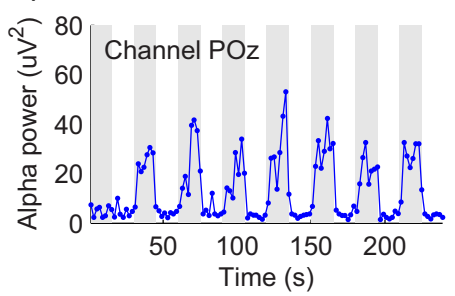

Subject 2
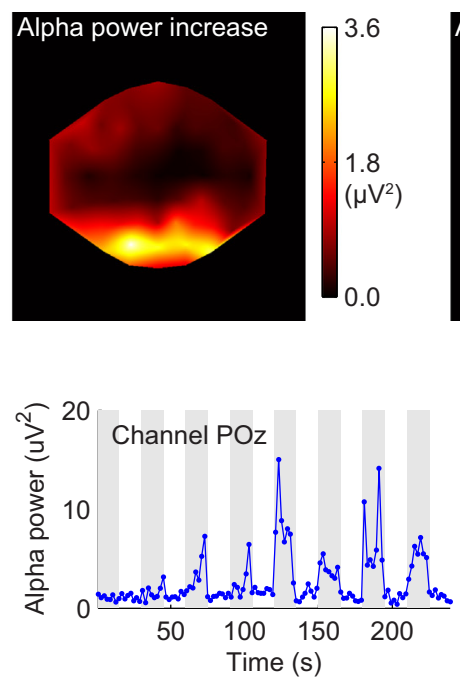

Subject 3
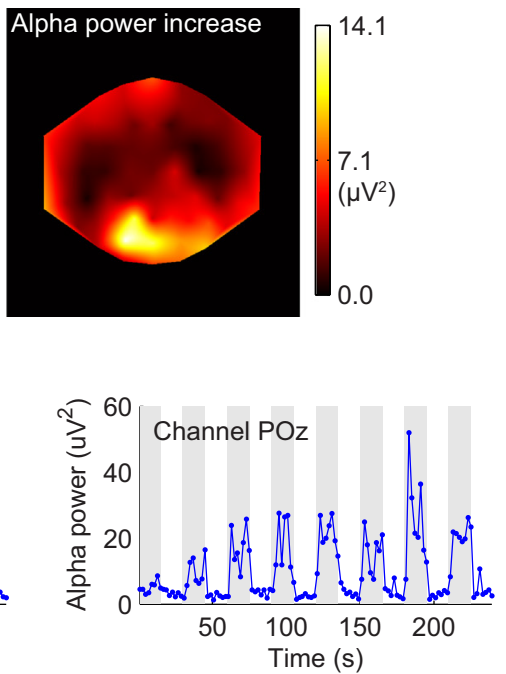

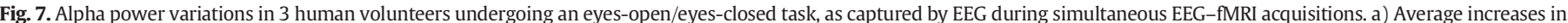

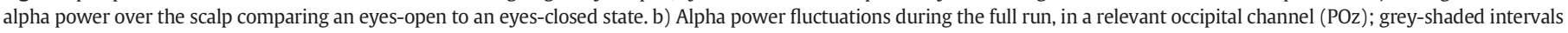

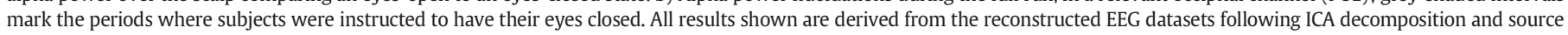
selection. 
a)

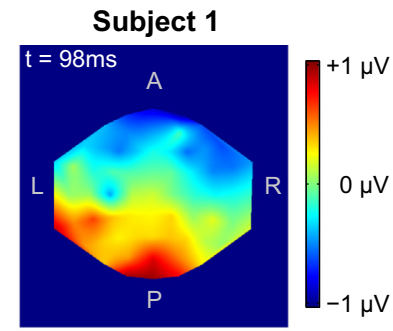

Subject 3

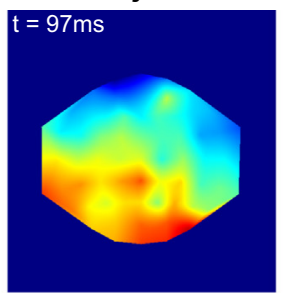

Subject 4

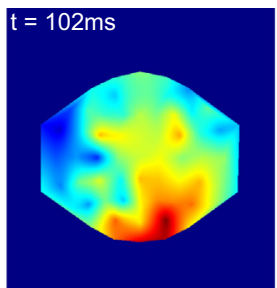

b)
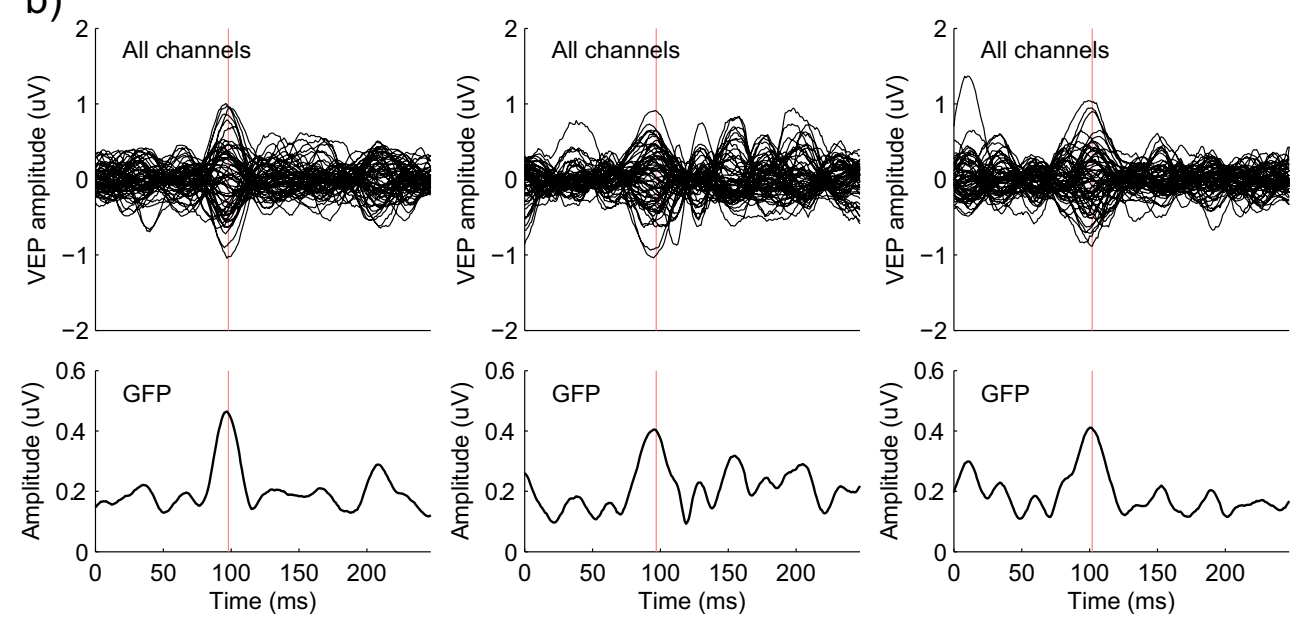

c)
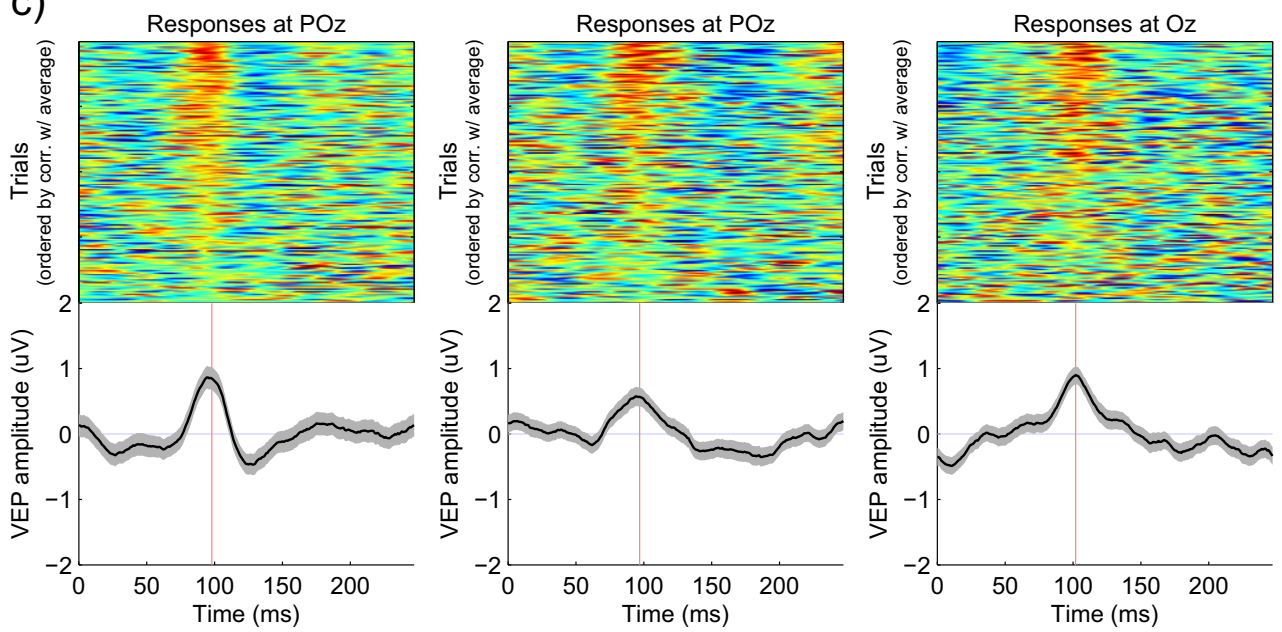

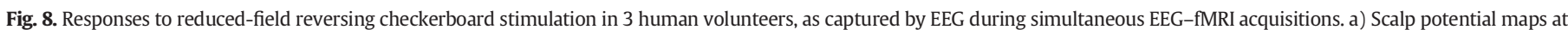

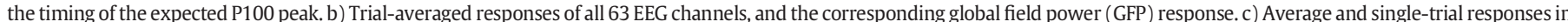

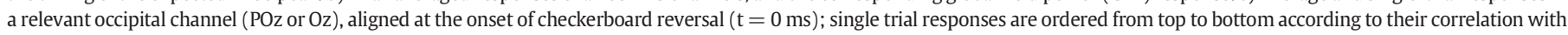

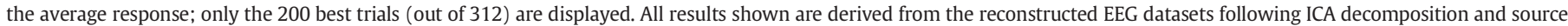
selection.

increasingly prohibitive as He availability decreases worldwide (Nuttall et al., 2012).

While initial developments have been presented to reduce vibrationrelated artifacts via post-acquisition data analysis (Rothlubbers et al., 2013), these approaches remain largely unexplored. Notably low coldhead-related contributions have been reported for a 9.4 T human scanner in which the coldheads are mounted on extended turrets and not directly on the magnet vessel (Neuner et al., 2013). While highly advantageous, this configuration is currently also rather unique. In this work, we directed our focus to the EEG acquisition system. The clear dependence of noise power on channel loop areas (Fig. 3) provided yet another indication that magnetic induction is the fundamental mechanism mediating coldhead-related noise propagation, and possibly of other environment sources. By reducing loop areas along the EEG transmission cables, significant improvements in signal quality were achieved through relatively simple modifications, which are inexpensive compared to the long-term costs of scanning with the coldheads switched off, or even modifying their placement. Furthermore, although not directly assessed in this work, gradient artifacts and a part of pulse artifacts are likewise strongly thought to be caused by magnetic induction effects on the EEG wire loops (Allen et al., 1998, 2000; Chowdhury et al., 2012; Mullinger et al., 2013). As such, cable bundling is also likely to have significantly reduced the impact of these important noise sources at the level of EEG transmission cables. The effects of cable shortening, while probably also favorable for pulse artifact reduction, are more complex for gradient artifacts, as longer cables may in some scanners reach a point where the 
a)

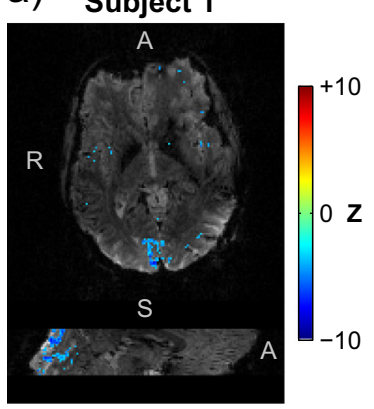

b)

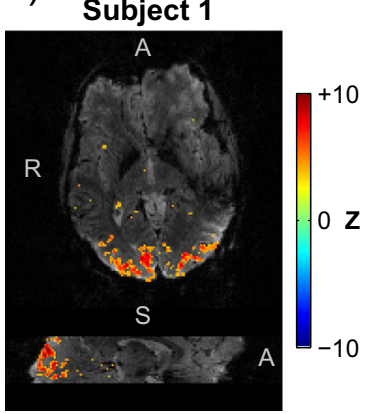

Subject 2

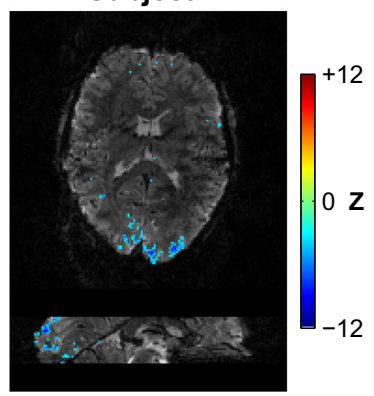

Subject 3

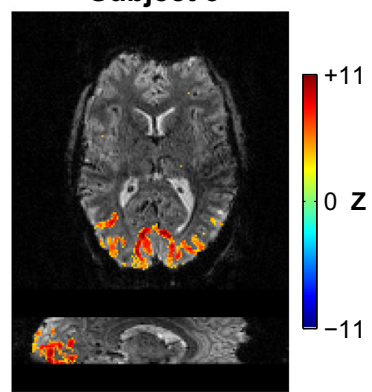

Subject 3

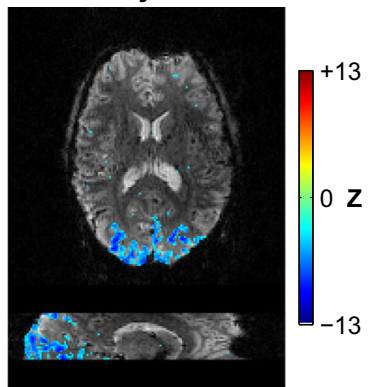

Subject 4

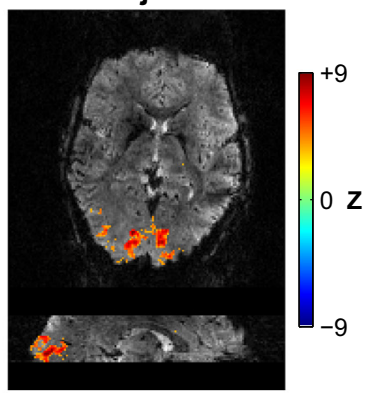

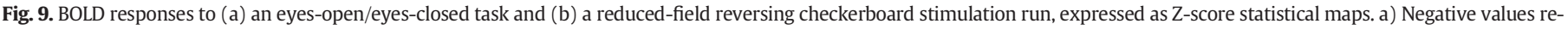

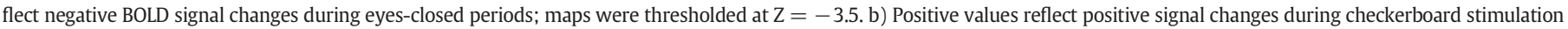
periods; maps were thresholded at $Z=+3.5$. Color bar ranges were manually restricted for clearer visualization.

gradient profile has become inverted, and thus benefit to some extent from flux cancellation effects.

Naturally, given the site-specificity of vibration-related noise contributions, the results obtained in study I cannot be directly translated to other EEG-fMRI setups, which may differ in coldhead configuration, scanner and patient bed architecture, $\mathrm{B}_{0}$ field distribution, and even surrounding equipment that may propagate vibrations to the scanner room. Likewise, the variations seen within this study are likely to have been affected not only by differences in cable length and geometry but also by properties such as stiffness and mass (for example, bundled cables tended to be stiffer than flat cables). This may explain some of the differences in spectral distribution (apart from overall power) observed for different cable configurations (Supplementary Fig. 1). Another aspect of great importance is the extent to which the EEG setup can actually be shortened without affecting the operation of the amplifiers. In this work, using shielded amplifiers and a head-only MRI system, equipped with short gradients and a Tx/Rx head RF array, it was possible to conduct simultaneous acquisitions at $7 \mathrm{~T}$ with the amplifiers placed just outside the RF coil, even with spin-echo EPI. While the $\mathrm{B}_{0}$ field extent does not significantly influence amplifier heating, and many imaging centers are equipped with head $\mathrm{Tx} / \mathrm{Rx}$ arrays for imaging, short gradients are less common and may play an important role in this setup-although the EEG amplifiers were actually positioned already inside the gradient region (Fig. 1). In general, it is likely that each particular EEG-MRI system configuration will require specific cable shortening tests prior to human studies, with gradual amplifier repositioning, or gradual increases in gradient slew-rates and RF power.

Finally, it is important to note that the noise reductions reported in the present study, while considerably large, are ascribed only to the contributions arising from the cables themselves. Loop areas formed by the leads on the cap surface remain at play and will still contribute to noise. Nonetheless, minimizing cable contributions is an important achievement by itself, as well as a potentially valuable step to improve the validity of various noise modeling and correction approaches already proposed in the literature, which focus mainly on the cap and assume negligible contributions from the following connection cables (Masterton et al., 2007; Yan et al., 2010).

\section{Safety and MRI data quality}

Given the interactions that can occur between EEG materials and RF (Lemieux et al., 1997), subject safety has always been a major point of concern with simultaneous EEG-fMRI (Laufs, 2012). While safety guidelines do exist, the pursuit of increasingly higher field strengths, higher EEG channel densities, and various custom modifications (coil designs, MR sequences), has continuously demanded site-specific safety assessments for setup validation. Temperature measurements in phantoms and humans have been extensively adopted for this purpose (Lemieux et al., 1997; Lazeyras et al., 2001; Mullinger et al., 2008a). While useful and practical, these tests are limited in spatial coverage, and cannot assess local SAR variations occurring in vivo within the brain. As a valuable complement, EM simulations using realistic head models allow the estimation of high-resolution SAR distributions across the head, but only a small number of studies have presented results from such approaches (Angelone et al., 2004, 2006).

In this study, we relied on both EM simulations and surface temperature measurements for safety assessment, with neither approach raising any significant concerns. The introduction of the EEG cap led to a small overall decrease in SAR, which is in fact contrasting with results from previous simulations conducted at $7 \mathrm{~T}$, reporting overall increases around 32\% (Angelone et al., 2006). This may be related to various differences existing between the two models, including electrode density, lead geometry, RF coil configuration, and the electromagnetic properties of the human model. In our particular implementation, a number of areas exhibiting SAR increases did appear with the introduction of the EEG cap (Fig. 5), notably in skin/skull regions, but the overall range of the SAR distribution remained practically unaltered. Regarding the temperature measurements, the fluctuations observed in EEG electrodes over the two 8 -minute acquisitions where below $1{ }^{\circ} \mathrm{C}$, in good agreement with previous reports at $7 \mathrm{~T}$ (Angelone et al., 2006; 
Mullinger et al., 2008a). Although our measurements were conducted over a relatively short period, compared to a more common duration of approximately $1 \mathrm{~h}$ for such sessions, the individual 8-minute runs were comparable to typical fMRI runs, and there were no reasons to expect any significant changes in the observed trends for subsequent runs. The EEG amplifiers did experience considerably larger temperature increases $\left(6.5^{\circ} \mathrm{C}\right.$ over $\left.16 \mathrm{~min}\right)$, although it was not possible to conclude how much of this heating was propagated from the RF gateway box (on which the amplifiers were standing and which by itself warms up during operation), or truly related to MR gradient or RF pulse effects. In any case, despite this increase, the observed values were still well within the normal operating range of the amplifiers $\left(10-40{ }^{\circ} \mathrm{C}\right)$, thus raising no cause for concern.

The functional (GE-EPI) and anatomical (GRE) images acquired for data quality assessment (Fig. 6) evinced artifacts caused by the introduction of the EEG system, notably an overall loss in spatial SNR and a few accentuated drops localized in superior regions. Temporal SNR losses were comparable to previous estimates at $7 \mathrm{~T}$ using a more standard EEG setup (Mullinger et al., 2008b), although here performed at a higher spatial resolution. Importantly, although significant, the losses observed in temporal SNR were considerably less severe than in spatial SNR, in good agreement with previous theoretical and experimental work (Luo and Glover, 2012), showing that functional information is in fact less affected by the introduction of EEG equipment than anatomical MR signals per se. Also in agreement with previous studies (Bonmassar et al., 2001; Lazeyras et al., 2001; Mullinger et al., 2008b), the $\mathrm{B}_{0}$ maps exhibited local susceptibility artifacts along the skin, likely coinciding with EEG electrodes, but focal enough not to extend into actual brain regions, which remained largely unaffected in terms of $B_{0}$ homogeneity. $\mathrm{B}_{1}^{+}$maps, on the other hand, displayed clear alterations both globally and in specific regions, which were largely coincident with the more accentuated local SNR drops observed in functional and anatomical images. Overall, the results obtained in this study strengthen the growing view that the properties of modern EEG caps have managed to limit susceptibility artifacts to a satisfactory level, even at ultra-high field (Krakow et al., 2000; Lazeyras et al., 2001). RF pulse disruption, in contrast, stands as an important degradation effect that can significantly reduce the available SNR, as well as compromise the performance of brain segmentation and other image processing steps (Mullinger et al., 2008b). $\mathrm{B}_{1}^{+}$inhomogeneity is already by itself a topic of intense research in ultra-high field MRI (Eggenschwiler et al., 2012). Aided by dedicated setup improvements, such as novel electrode and lead materials (Vasios et al., 2006), it is likely that EEG-related $\mathrm{B}_{1}^{+}$degradation can be satisfactorily reduced in the near future.

\section{Simultaneous EEG-fMRI acquisitions}

In addition to this work, only a handful of studies so far have conducted simultaneous EEG-fMRI acquisitions in humans above $4 \mathrm{~T}$ (Vasios et al., 2006; Mullinger et al., 2008a; Brookes et al., 2009). Following prior safety assessments on phantom and numerical models, human recordings proceeded without any indication of heating. Clear average EEG responses were observed for most subjects over both eyes-open/ closed and VEP runs, coherent with the patterns expected for the respective paradigms (Pfurtscheller and Lopes da Silva, 1999; Skrandies, 2005).

At a single-trial level, response detection sensitivity was considerably different for the two runs: in the eyes-open/closed task, alpha power variations could be clearly observed over most blocks in 3 of the 4 subjects, while for the VEP run only approximately half of the trials in each run/subject exhibited statistically significant responses. These discrepancies in sensitivity may be related to the nature of the elicited responses: alpha power modulation via eyes-closing tasks is known to be a strong and robust effect, which has been successfully observed at fields up to $9.4 \mathrm{~T}$ (Neuner et al., 2014). In contrast, the checkerboard stimuli used in this study were presented with a reduced FOV, likely to elicit weaker responses which are harder to decouple from artifacts and ongoing neuronal activity (ACNS, 2006). On the other hand, alpha fluctuations occur at a frequency range $(8-12 \mathrm{~Hz})$ which was less contaminated by gradient and pulse artifact contributions, a factor which may have also contributed to this difference. Also worthy of note, visual stimulation in the VEP paradigm was phase-locked with slice acquisition every 25 slices, rendering gradient artifact correction potentially more susceptible to remove VEP signal. This was addressed with a fairly EEG-conservative AAS approach, using fixed gaps between averaged slices of $400 \mathrm{~ms}$ to mitigate the reduction of correlated EEG activity (Niazy et al., 2005), combined with random jittering to reduce phase locking with the VEP. The adopted spacing in turn required averaging over relatively large time windows (approximately $20 \mathrm{~s}$ ), leading to a certain compromise in adaptability to changes in the artifact profile. This limitation was potentially relevant in cases where the artifact was less stationary, such as due to frequent subject motion, although artifact residuals were still further reduced by OBS and temporal bandpass filtering, and were not found to be problematic in these data.

It is also important to note that the use of ICA for denoising in these datasets, while undeniably valuable, can be compromised by the fact that motion artifacts, including residual pulse artifacts and spontaneous subject movements, are not truly stationary sources, especially at high field (Debener et al., 2008), and thus may not be adequately separable from true neuronal sources. Given the importance of response sensitivity at a single-trial level for simultaneous EEG-fMRI, it is desirable to further explore this question in future work, for example by comparing responses to checkerboards of different FOV/contrast, and exploring alternative denoising techniques such as iterative ICA (Iyer and Zouridakis, 2007), wavelet-based approaches (Quian Quiroga and Garcia, 2003), or beamformer methodologies (Brookes et al., 2009).

Data from fMRI acquisitions exhibited clear responses in both functional runs for all but one subject, with significant paradigm-related signal changes arising, as expected, in visual areas (Fig. 9). In the eyesopen/closed run, the 4th subject showed neither task-related alpha power variations nor BOLD signal changes, suggesting non-compliance with the task. In general, the robustness of the elicited responses suggests that the $\mathrm{B}_{1}^{+}$disruption effects observed in study II, while clearly reducing image SNR in the parietal lobe, did not hinder BOLD sensitivity in the occipital cortex. This discrepancy may be due to the distinct spatial localization of the two regions, but may also be related to the inherent differences between spatial SNR and functional sensitivity (temporal SNR), as previously mentioned, especially given the importance of signal-dependent physiological noise contributions at higher fields. In any case, this outcome follows the trend observed in various other studies at lower fields, with diverse types of stimuli, reporting little to no effects of the presence of the EEG system on BOLD sensitivity (Bonmassar et al., 2001; Lazeyras et al., 2001; Luo and Glover, 2012).

\section{Conclusion}

The results obtained in this work demonstrate important benefits of careful optimization of the EEG signal chain for simultaneous EEG-fMRI. Focusing on the transmission stage between the EEG cap and amplifiers, we have confirmed that both cable shortening and bundling effectively help reducing cable noise contributions to large extents. Under the conditions of typical functional acquisitions, temperature measurements and EM simulations did not raise any significant safety concerns for the optimized setup. Regarding MRI data quality, we conclude that $\mathrm{B}_{1}^{+}$ field disruption is currently the main cause of image degradation due to EEG materials at $7 \mathrm{~T}$, with susceptibility artifacts exerting only minor effects in actual brain regions. Based on human recordings performed under eyes-open/closed tasks and checkerboard stimulation, we conclude that alpha-wave modulation, VEPs and the concomitant BOLD signal changes can be detected with favorable sensitivity. Overall, setup improvements such as those proposed in this work, together with denoising approaches specifically tailored for simultaneous EEG-fMRI, 
steadily aid to bring this multimodal approach to satisfactory standards of signal quality, robustness and sensitivity, allowing for the full exploit of the benefits offered by high-field imaging.

Supplementary data to this article can be found online at http://dx. doi.org/10.1016/j.neuroimage.2014.10.055.

\section{Acknowledgments}

This work was supported by Centre d'Imagerie BioMédicale (CIBM) of the UNIL, UNIGE, HUG, CHUV, EPFL and the Leenaards and Jeantet Foundations, and by the Portuguese Science Foundation (FCT) through grants SFRH/BD/51449/2011, PTDC/SAU-ENB/112294/2009 and PEstOE/EEI/LA0009/2011.

\section{References}

ACNS, 2006. Guideline 9B: guidelines on visual evoked potentials. J. Clin. Neurophysiol 138-156.

Allen, P.J., Polizzi, G., Krakow, K., Fish, D.R., Lemieux, L., 1998. Identification of EEG events in the MR scanner: the problem of pulse artifact and a method for its subtraction. Neurolmage 8, 229-239.

Allen, P.J., Josephs, O., Turner, R., 2000. A method for removing imaging artifact from continuous EEG recorded during functional MRI. NeuroImage 12, 230-239.

Angelone, L.M., Potthast, A., Segonne, F., Iwaki, S., Belliveau, J.W., Bonmassar, G., 2004 Metallic electrodes and leads in simultaneous EEG-MRI: specific absorption rate (SAR) simulation studies. Bioelectromagnetics 25, 285-295.

Angelone, L.M., Vasios, C.E., Wiggins, G., Purdon, P.L., Bonmassar, G., 2006. On the effect of resistive EEG electrodes and leads during 7 T MRI: simulation and temperature measurement studies. Magn. Reson. Imaging 24, 801-812.

Arrubla, J., Neuner, I., Hahn, D., Boers, F., Shah, N.J., 2013. Recording visual evoked potentials and auditory evoked P300 at 9.4 T static magnetic field. PLoS ONE 8, e62915.

Assecondi, S., Ferrari, P., Jovicich, J., 2013. A compact setup to improve the quality of EEG data recorded during fMRI. 21st Annual Meeting \& Exhibition of the International Society for Magnetic Resonance in Medicine, Salt Lake City, Utah, USA.

Boly, M., Balteau, E., Schnakers, C., Degueldre, C., Moonen, G., Luxen, A., Phillips, C. Peigneux, P., Maquet, P., Laureys, S., 2007. Baseline brain activity fluctuations predict somatosensory perception in humans. Proc. Natl. Acad. Sci. U. S. A. 104, 12187-12192.

Bonmassar, G., Anami, K., Ives, J., Belliveau, J.W., 1999. Visual evoked potential (VEP) measured by simultaneous 64-channel EEG and 3 T fMRI. Neuroreport 10, 1893-1897.

Bonmassar, G., Hadjikhani, N., Ives, J.R., Hinton, D., Belliveau, J.W., 2001. Influence of EEC electrodes on the BOLD fMRI signal. Hum. Brain Mapp. 14, 108-115.

Britz, J., Van De Ville, D., Michel, C.M., 2010. BOLD correlates of EEG topography reveal rapid resting-state network dynamics. NeuroImage 52, 1162-1170.

Brookes, M.J., Vrba, J., Mullinger, K.J., Geirsdottir, G.B., Yan, W.X., Stevenson, C.M., Bowtell R., Morris, P.G., 2009. Source localisation in concurrent EEG/fMRI: applications at $7 \mathrm{~T}$. NeuroImage 45, 440-452.

Chowdhury, M., Mullinger, K., Bowtell, R., 2012. Simultaneous EEG-fMRI: evaluating the effect of the cabling configuration on the gradient artefact. 20th Annual Meeting \& Exhibition of the International Society for Magnetic Resonance in Medicine Melbourne, Australia.

Christ, A., Kainz, W., Hahn, E.G., Honegger, K., Zefferer, M., Neufeld, E., Rascher, W., Janka, R., Bautz, W., Chen, J., Kiefer, B., Schmitt, P., Hollenbach, H.P., Shen, J., Oberle, M. Szczerba, D., Kam, A., Guag, J.W., Kuster, N., 2010. The virtual family-development of surface-based anatomical models of two adults and two children for dosimetric simulations. Phys. Med. Biol. 55, N23-N38.

Debener, S., Kranczioch, C., Herrmann, C.S., Engel, A.K., 2002. Auditory novelty oddball allows reliable distinction of top-down and bottom-up processes of attention. Int. J. Psychophysiol. 46, 77-84

Debener, S., Mullinger, K.J., Niazy, R.K., Bowtell, R.W., 2008. Properties of the ballistocardiogram artefact as revealed by EEG recordings at 1.5, 3 and $7 \mathrm{~T}$ static magnetic field strength. Int. J. Psychophysiol. 67, 189-199.

Deelchand, D.K., Van de Moortele, P.F., Adriany, G., Iltis, I., Andersen, P., Strupp, J.P., Vaughan, J.T., Ugurbil, K., Henry, P.G., 2010. In vivo 1H NMR spectroscopy of the human brain at 9.4 T: initial results. J. Magn. Reson. 206, 74-80.

Dempsey, M.F., Condon, B., 2001. Thermal injuries associated with MRI. Clin. Radiol. 56, 457-465.

Dempsey, M.F., Condon, B., Hadley, D.M., 2001. Investigation of the factors responsible for burns during MRI. J. Magn. Reson. Imaging 13, 627-631.

Duyn, J.H., 2012. The future of ultra-high field MRI and fMRI for study of the human brain. Neurolmage 62, 1241-1248.

Eggenschwiler, F., Kober, T., Magill, A.W., Gruetter, R., Marques, J.P., 2012. SA2RAGE: new sequence for fast B1+-mapping. Magn. Reson. Med. 67, 1609-1619.

Goldman, R.I., Stern, J.M., Engel Jr., J., Cohen, M.S., 2002. Simultaneous EEG and fMRI of the alpha rhythm. Neuroreport 13, 2487-2492.

Gotman, J., Pittau, F., 2011. Combining EEG and fMRI in the study of epileptic discharges. Epilepsia 52 (Suppl. 4), 38-42.

Grouiller, F., Vercueil, L., Krainik, A., Segebarth, C., Kahane, P., David, O., 2007. A comparative study of different artefact removal algorithms for EEG signals acquired during functional MRI. NeuroImage 38, 124-137.
Iyer, D., Zouridakis, G., 2007. Single-trial evoked potential estimation: comparison between independent component analysis and wavelet denoising. Clin. Neurophysiol. $118,495-504$.

Jorge, J., van der Zwaag, W., Figueiredo, P., 2014. EEG-fMRI integration for the study of human brain function. NeuroImage 102 (1), 24-34

Krakow, K., Allen, P.J., Symms, M.R., Lemieux, L., Josephs, O., Fish, D.R., 2000. EEG recording during fMRI experiments: image quality. Hum. Brain Mapp. 10, 10-15.

Laufs, H., 2012. A personalized history of EEG-fMRI integration. NeuroImage 62, 1056-1067.

Lazeyras, F., Zimine, I., Blanke, O., Perrig, S.H., Seeck, M., 2001. Functional MRI with simultaneous EEG recording: feasibility and application to motor and visual activation. J. Magn. Reson. Imaging 13, 943-948.

Lemieux, L., Allen, P.J., Franconi, F., Symms, M.R., Fish, D.R., 1997. Recording of EEG during fMRI experiments: patient safety. Magn. Reson. Med. 38, 943-952.

Luo, Q., Glover, G.H., 2012. Influence of dense-array EEG cap on fMRI signal. Magn. Reson. Med. 68, 807-815.

Mahajan, Y., McArthur, G., 2012. Maturation of visual evoked potentials across adolescence. Brain Dev. 34, 655-666.

Masterton, R.A., Abbott, D.F., Fleming, S.W., Jackson, G.D., 2007. Measurement and reduction of motion and ballistocardiogram artefacts from simultaneous EEG and fMRI recordings. Neurolmage 37, 202-211.

Mullinger, K., Bowtell, R., 2011. Combining EEG and fMRI. Methods Mol. Biol. 711, 303-326.

Mullinger, K., Brookes, M., Stevenson, C., Morgan, P., Bowtell, R., 2008a. Exploring the feasibility of simultaneous electroencephalography/functional magnetic resonance imaging at 7 T. Magn. Reson. Imaging 26, 968-977.

Mullinger, K., Debener, S., Coxon, R., Bowtell, R., 2008b. Effects of simultaneous EEG recording on MRI data quality at 1.5, 3 and 7 tesla. Int. J. Psychophysiol. 67, 178-188.

Mullinger, K.J., Havenhand, J., Bowtell, R., 2013. Identifying the sources of the pulse artefact in EEG recordings made inside an MR scanner. NeuroImage 71, 75-83.

Neuner, I., Warbrick, T., Arrubla, J., Felder, J., Celik, A., Reske, M., Boers, F., Shah, N.J., 2013. EEG acquisition in ultra-high static magnetic fields up to $9.4 \mathrm{~T}$. Neurolmage 68, 214-220.

Neuner, I., Arrubla, J., Felder, J., Shah, N.J., 2014b. Simultaneous EEG-fMRI acquisition at low, high and ultra-high magnetic fields up to $9.4 \mathrm{~T}$ : perspectives and challenges. Neurolmage 102 (1), 71-79.

Niazy, R.K., Beckmann, C.F., Iannetti, G.D., Brady, J.M., Smith, S.M., 2005. Removal of FMRI environment artifacts from EEG data using optimal basis sets. NeuroImage 28, 720-737.

Niedermeyer, E., Lopes da Silva, F.H., 2005. Electroencephalography: Basic Principles, Clinical Applications, and Related Fields, 5th ed. Lippincott Williams \& Wilkins, Philadelphia.

Nierhaus, T., Gundlach, C., Goltz, D., Thiel, S.D., Pleger, B., Villringer, A., 2013. Internal ventilation system of MR scanners induces specific EEG artifact during simultaneous EEG-fMRI. NeuroImage 74, 70-76.

Noth, U., Laufs, H., Stoermer, R., Deichmann, R., 2012. Simultaneous electroencephalographyfunctional MRI at $3 \mathrm{~T}$ : an analysis of safety risks imposed by performing anatomical reference scans with the EEG equipment in place. J. Magn. Reson. Imaging 35, 561-571.

Novitski, N., Anourova, I., Martinkauppi, S., Aronen, H.J., Naatanen, R., Carlson, S., 2003. Effects of noise from functional magnetic resonance imaging on auditory event-related potentials in working memory task. Neurolmage 20, 1320-1328.

Nuttall, W.J., Clarke, R.H., Glowacki, B.A., 2012. Resources: stop squandering helium. Nature $485,573-575$.

Ogawa, S., Lee, T.M., Kay, A.R., Tank, D.W., 1990. Brain magnetic resonance imaging with contrast dependent on blood oxygenation. Proc. Natl. Acad. Sci. U. S. A. 87, 9868-9872.

Pfurtscheller, G., Lopes da Silva, F.H. 1999. Event-related EEG/MEG synchronization and desynchronization: basic principles. Clin. Neurophysiol. 110, 1842-1857.

Quian Quiroga, R., Garcia, H., 2003. Single-trial event-related potentials with wavelet denoising. Clin. Neurophysiol. 114, 376-390.

Ritter, P., Becker, R., Freyer, F., Villringer, A., 2010. EEG Quality: The Image Acquisition Artefact. In: Mulert, C., Lemieux, L. (Eds.), EEG-fMRI. Springer, Berlin Heidelberg, pp. 153-171.

Rothlubbers, S, Relvas, V Leal, A Figueiredo, P. 2013. Reduction of EEG artefacts induced by vibration in the MR-environment. Conf Proc IEEE Eng Med Biol Soc 2013, pp. 2092-2095.

Scheeringa, R., Fries, P., Petersson, K.M., Oostenveld, R., Grothe, I., Norris, D.G., Hagoort, P., Bastiaansen, M.C. 2011. Neuronal dynamics underlying high- and low-frequency EEG oscillations contribute independently to the human BOLD signal. Neuron 69, 572-583.

Seeck, M., Lazeyras, F., Michel, C.M., Blanke, O., Gericke, C.A., Ives, J., Delavelle, J., Golay, X., Haenggeli, C.A., de Tribolet, N., Landis, T., 1998. Non-invasive epileptic focus localization using EEG-triggered functional MRI and electromagnetic tomography. Electroencephalogr. Clin. Neurophysiol. 106, 508-512.

Skrandies, W., 2005. Brain mapping of visual evoked activity-topographical and functional components. Acta Neurol. Taiwan 14, 164-178.

Smith, S.M., Jenkinson, M., Woolrich, M.W., Beckmann, C.F., Behrens, T.E., Johansen-Berg, H., Bannister, P.R., De Luca, M., Drobnjak, I., Flitney, D.E., Niazy, R.K., Saunders, J., Vickers, J., Zhang, Y., De Stefano, N., Brady, J.M., Matthews, P.M., 2004. Advances in functional and structural MR image analysis and implementation as FSL. Neurolmage 23 (Suppl. 1), S208-S219.

Triantafyllou, C., Hoge, R.D., Krueger, G., Wiggins, C.J., Potthast, A., Wiggins, G.C., Wald, L.L., 2005. Comparison of physiological noise at $1.5 \mathrm{~T}, 3 \mathrm{~T}$ and $7 \mathrm{~T}$ and optimization of fMRI acquisition parameters. Neurolmage 26, 243-250.

Turner, R., Jezzard, P., Wen, H., Kwong, K.K., Le Bihan, D., Zeffiro, T., Balaban, R.S., 1993. Functional mapping of the human visual cortex at 4 and 1.5 tesla using deoxygenation contrast EPI. Magn. Reson. Med. 29, 277-279. 
van der Zwaag, W., Francis, S., Head, K., Peters, A., Gowland, P., Morris, P., Bowtell, R. 2009. fMRI at 1.5, 3 and 7 T: characterising BOLD signal changes. NeuroImage 47, 1425-1434.

Vasios, C.E., Angelone, L.M., Purdon, P.L., Ahveninen, J., Belliveau, J.W., Bonmassar, G., 2006. EEG/(f)MRI measurements at 7 Tesla using a new EEG cap ("InkCap"). Neurolmage 33, 1082-1092.

Worsley, K.J., Friston, K.J., 1995. Analysis of fMRI time-series revisited-again. Neurolmage 2, 173-181.
Yacoub, E., Harel, N., Ugurbil, K., 2008. High-field fMRI unveils orientation columns in humans. Proc. Natl. Acad. Sci. U. S. A. 105, 10607-10612.

Yan, W.X., Mullinger, K.J., Brookes, M.J., Bowtell, R., 2009. Understanding gradient artefacts in simultaneous EEG/fMRI. NeuroImage 46, 459-471.

Yan, W.X., Mullinger, K.J., Geirsdottir, G.B., Bowtell, R., 2010. Physical modeling of pulse artefact sources in simultaneous EEG/fMRI. Hum. Brain Mapp. 31, 604-620. 